\title{
Article \\ Single-Shot On-Axis Fizeau Polarization Phase-Shifting Digital Holography for Complex-Valued Dynamic Object Imaging
}

\author{
Hanzi Liu, Vinu R. V. (D, Hongliang Ren, Xingpeng Du, Ziyang Chen *®) and Jixiong Pu \\ Fujian Key Laboratory of Light Propagation and Transformation, College of Information Science and Engineering, \\ Huaqiao University, Xiamen 361021, China; 20013082008@stu.hqu.edu.cn (H.L.); vinurv@hqu.edu.cn (V.R.V.); \\ renhongliang@ustc.edu (H.R.); 1915111006@stu.hqu.edu.cn (X.D.); jixiong@hqu.edu.cn (J.P.) \\ * Correspondence: ziyang@hqu.edu.cn
}

check for updates

Citation: Liu, H.; R. V., V.; Ren, H.; $\mathrm{Du}, \mathrm{X} . ;$ On-Axis Fizeau Polarization

Phase-Shifting Digital Holography for Complex-Valued Dynamic Object Imaging. Photonics 2022, 9, 126. https://doi.org/10.3390/ photonics 9030126

Received: 17 January 2022

Accepted: 14 February 2022

Published: 23 February 2022

Publisher's Note: MDPI stays neutral with regard to jurisdictional claims in published maps and institutional affiliations.

Copyright: (C) 2022 by the authors. Licensee MDPI, Basel, Switzerland. This article is an open access article distributed under the terms and conditions of the Creative Commons Attribution (CC BY) license (https:// creativecommons.org/licenses/by/ $4.0 /)$.

\begin{abstract}
Digital holography assisted with inline phase-shifting methods has the benefit of a large field of view and a high resolution, but it is limited in dynamic imaging due to sequential detection of multiple holograms. Here we propose and experimentally demonstrate a single-shot phase-shifting digital holography system based on a highly stable on-axis Fizeau-type polarization interferometry. The compact on-axis design of the system with the capability of instantaneous recording of multiple phase-shifted holograms and with robust stability features makes the technique a novel tool for the imaging of complex-valued dynamic objects. The efficacy of the approach is demonstrated experimentally by complex field imaging of various kinds of reflecting-type static and dynamic objects. Moreover, a quantitative analysis on the robust phase stability and sensitivity of the technique is evaluated by comparing the approach with conventional phase-shifting methods. The high phase stability and dynamic imaging potential of the technique are expected to make the system an ideal tool for quantitative phase imaging and real-time imaging of dynamic samples.
\end{abstract}

Keywords: digital holography; interferometry; phase-shifting; polarization; complex field imaging; quantitative phase imaging

\section{Introduction}

The applied domains of digital holography (DH) have an urge of interest in recent times with advancements in high resolution image sensors and modulators with potential applications in the areas of interferometry, microscopy, quantitative phase imaging (QPI), three-dimensional (3D) imaging, ultra-fast imaging, imaging through scattering medium, ghost imaging, etc. [1-8]. DH can achieve the demand of simultaneous imaging or of the characterization of multidimensional information, such as 3D structure, amplitude, phase, polarization, etc., by making use of either inline or off-axis DH based approaches [9-13]. However, the quality of image restoration in holographic techniques suffers from undesirable zero order and twin image occupancy in the hologram. The evolution of computational techniques in the last two decades, with the introduction of phase retrieval algorithms [14-16] and machine learning [17,18] approaches, brings forth the high-quality imaging in $\mathrm{DH}$, but it may be limited in some imaging scenarios due to the phase convergence issue, computational time, learning mechanisms, etc. Alternatively, the integration of phase-shifting in $\mathrm{DH}$ has prominent advantages, as the reconstructed image is free from the undesired terms of zero order and from conjugate terms with the cost of a sequential multiple recording of the phase-shifted holograms for the reliable reconstruction of the image $[19,20]$. Thus, the phase-shifting approaches permit the implementation of an inline geometry with full space-bandwidth utilization of the camera, and thereby provide a large field of view and a high spatial resolution to the imaging system. Usually, the sequential phase modulation is achieved by the utilization of piezoelectric mirrors, spatial light modulators, rotating retarders, acousto-optic or electro-optic modulators, etc. Although 
phase-shifting digital holography (PSDH) has its full potential in 3D complex field image reconstruction, the sequential multiple recording of the holograms limits the execution of the system in dynamic object imaging.

Owing to the dynamic imaging challenge in PSDH techniques, a parallel-PSDH technique, with the potential feature of instantaneous recording of the multiple phase-shifted holograms, is demonstrated by using the phase-shifting array device in the reference arm of the holography system [21,22]. Later, the parallel phase-shifting approaches were extended to high-speed imaging with the utilization of sophisticated spatial light modulators [23,24] and a polarized camera [24]. Subsequently, the effectiveness of the polarized camera in combination with phase-shifting approaches was demonstrated by developing a simultaneous polarization Mirau interferometer [25], by the imaging of flow and sound [26,27], by a snapshot diffraction microscope [28], etc. In addition, the potential of parallel phase-shifting techniques with polarized image sensors is exploited to develop a single-shot incoherent digital holography system and to develop a further extension to microscopy [29-31]. Later, the imaging of dynamic objects with parallel phase-shifting approaches was demonstrated with the help of two-channel holography schemes [32,33], dual polarization imaging cameras [34], etc. However, the two-channel configurations may suffer from spatial and temporal phase stability issues due to external vibrations in the medium and due to the aberrations in the optical components as the beam propagation occurs through a different optical path.

In the present work, we propose and experimentally demonstrate a single-shot on-axis phase-shifting digital holography system that utilizes a Fizeau-type polarization interferometry technique for complex-valued dynamic object imaging. The developed system relies on the Fizeau-type interferometry scheme, in which the orthogonal polarization components for the object and reference beams were facilitated by using a wire grid polarizer (WGP). The technique makes use of the parallel phase-shifting approach with space division multiplexing for the development of high-speed instantaneous recording of multiple phase-shifted holograms. This is realized by using the combination of a quarter wave plate (QWP) and a micro polarizer array with different orientations encoded in the polarized camera to detect polarized light fields from the Fizeau polarization system. The compact on-axis design makes the system robust to external vibrations and provides high spatial and temporal stability to the holography scheme. Furthermore, the applicability of the proposed technique is experimentally demonstrated for simultaneous complex field imaging of various static and dynamic complex-valued objects. Additionally, a quantitative analysis is performed to evaluate the phase stability and sensitivity of the system in comparison with conventional phase-shifting methods.

\section{Principles and Methods}

\subsection{Fizeau Polarization Phase-Shifting Digital Holography (FP-PSDH)}

The FP-PSDH system makes use of a highly stable Fizeau-polarization interferometry scheme for the generation of on-axis near common-path propagating orthogonal reference and object beams. The compact on-axis design of the polarization interferometry technique is facilitated by employing a wire grid polarizer (WGP) consisting of an array of metallic wires. A conceptual schematic of the orthogonal polarized beam generation with a WGP is represented in Figure 1a. The illumination of a linearly $45^{\circ}$ polarized beam relative to the wire grid on the WGP results in a reflected beam with the polarization component parallel to the direction of the wire grid (s-polarized) and in a transmitted beam with the polarization component perpendicular to the wire grid (p-polarized). In our proposed scheme, the s-polarized component serves as the reference beam, and the p-polarized component serves as the object beam for polarization phase-shifting. The back-scattered p-polarized object beam propagates along with the reference beam in a common path 
after interacting with the object. The resulting common-path propagating light fields are given by,

$$
\begin{aligned}
E_{p}(\hat{\boldsymbol{r}}) & =A_{p}(\hat{\boldsymbol{r}}) \exp \left(i \phi_{p}(\hat{\boldsymbol{r}})\right) \\
E_{S}(\hat{\boldsymbol{r}}) & =A_{\mathcal{S}}(\hat{\boldsymbol{r}}) \exp \left(i \phi_{s}(\hat{\boldsymbol{r}})\right)
\end{aligned}
$$

where $A_{p}(\hat{r})$ and $A_{s}(\hat{\boldsymbol{r}})$ are the amplitude information, and where $\phi_{p}(\hat{\boldsymbol{r}})$ and $\phi_{s}(\hat{\boldsymbol{r}})$ are the phase information of the object modulated p-polarized and reference s-polarized beams, respectively.

(a)

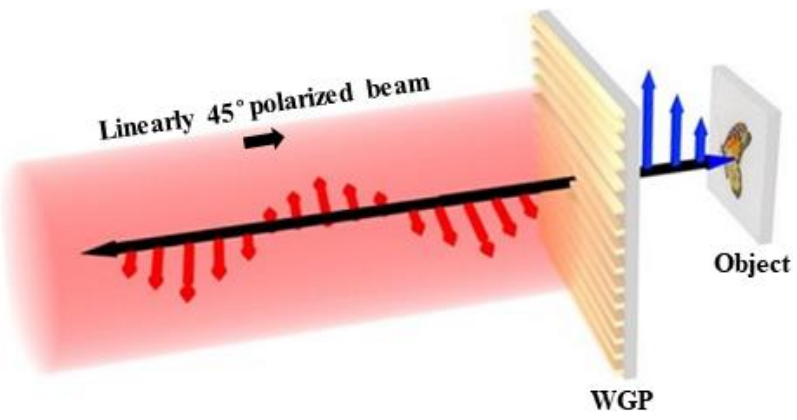

(b)

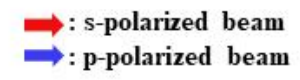

WGP

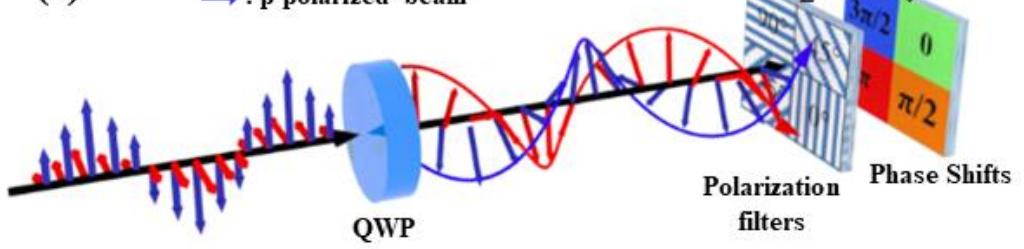

Figure 1. (a) Conceptual schematic of orthogonal polarized beam generation with the wire grid polarizer (WGP); (b) Schematic of the space division multiplexing mechanism with QWP and polarization filters in the polarized camera for single-shot detection of multiple phase-shifted holograms.

To implement a single-shot recording of the multiple phase-shifting holograms, we utilized the space division multiplexing technique with the combination of a QWP and a polarized camera [29]. A conceptual schematic of the multiplexing implementation in the proposed approach is demonstrated in Figure $1 \mathrm{~b}$. The on-axis propagating reference and object beams passed through the QWP with its fast axis at $45^{\circ}$ with respect to the common-path propagating polarized beams, thereby changing the polarization state to the respective circularly polarized beams and reaching the polarized camera sensor plane. The camera sensor comprises unique nano-wire grid arrays with four separate polarizing filters angled at $0^{\circ}, 45^{\circ}, 90^{\circ}$, and $135^{\circ}$ positioned in a systematic pattern across the sensor. The electric field components of the circularly polarized beams at the exit plane of the polarizing filters of the sensor plane are given by,

$$
E_{k}(\boldsymbol{r})=\frac{1}{\sqrt{2}}\left(\begin{array}{cc}
\cos ^{2} \theta & \cos \theta \sin \theta \\
\cos \theta \sin \theta & \sin ^{2} \theta
\end{array}\right)\left(\begin{array}{ll}
1 & i \\
i & 1
\end{array}\right) E_{k}(\hat{\boldsymbol{r}})
$$

where $k=p$ or $s$, the matrices represent the Jones matrices corresponding to the polarizer filters at $\theta$ orientations and the QWP with its fast axis at $45^{\circ}$, respectively, and $E_{k}(\hat{r})$ represents the $p$ or $s$ polarized beams with the respective Jones representation. Therefore, the intensity distribution $I(\boldsymbol{r})=E_{k}^{*}(\boldsymbol{r}) E_{k}(\boldsymbol{r})$ at the sensor plane is expressed by,

$$
I(\boldsymbol{r})=\frac{1}{2}\left[A_{p}^{2}(\boldsymbol{r})+A_{s}^{2}(\boldsymbol{r})+2 A_{p}(\boldsymbol{r}) A_{s}(\boldsymbol{r}) \cos (2 \theta-\Delta \phi(\boldsymbol{r}))\right]
$$

where $\Delta \phi(\boldsymbol{r})=\phi_{p}(\boldsymbol{r})-\phi_{s}(\boldsymbol{r})$ is the phase difference between the object and the reference field at the sensor plane. The space-division multiplexing, resulting from the interaction of circularly polarized object and reference beams with the polarization filters at specific 
angles, produces four phase-shifting holograms: $I(\boldsymbol{r} ; 0), I(\boldsymbol{r} ; \pi / 2), I(\boldsymbol{r} ; \pi) \& I(\boldsymbol{r} ; 3 \pi / 2)$ with $\pi / 2$ phase-shift represented in the conceptual schematic of Figure 1b. The single-shot detection of the multiple phase-shifted holograms at the sensor plane provides the flexible advantage of extraction of the complex field distribution of the object modulated information using the conventional phase-shifting interferometry relation, which is expressed as,

$$
E(\boldsymbol{r})=(I(\boldsymbol{r} ; 0)-I(\boldsymbol{r} ; \pi))+i(I(\boldsymbol{r} ; 3 \pi / 2)-I(\boldsymbol{r} ; \pi / 2))
$$

The retrieval of the complex field distribution at the sensor plane gives the provision for the recovery of the amplitude and phase distribution of the complex-valued object at any arbitrary plane by using the digital backpropagation approaches [35].

\subsection{Experimental Design}

A schematic sketch of the experimental design of FP-PSDH is shown in Figure 2. A vertically polarized He-Ne laser source (CVI Melles Griot-25-LHP-928-230) of wavelength $632.8 \mathrm{~nm}$, which is converted in to a linearly $45^{\circ}$ polarized by a half wave plate (HWP), acts as the source beam for the proposed Fizeau polarization system. The beam is spatially filtered and collimated to generate a uniform beam with a plane wavefront. The transmitted beam from a non-polarizing beam splitter (BS) illuminates the WGP (ThorLab-WP50L-VIS) and produces two orthogonal polarization components from the input polarized beam, where the reflected beam (s-polarized) acts as the reference beam and where the transmitted beam (p-polarized) acts as the object beam for the Fizeau polarization interferometry. The p-polarized beam interacts with the desired object, and the backscattered object beam transmits again through the WGP and propagates in an on-axis common-path with the s-polarized beam. The object and the reference beam from the WGP propagate in an on-axis geometry and are reflected from the BS. These on-axis propagated polarized light fields transmit through the QWP with their fast axis oriented at $45^{\circ}$ before reaching the monochrome polarization camera. The camera is 5.1 megapixels with a Sony IMX250MZR CMOS polarized sensor (active pixels $2464 \times 2056$ with pixel size $3.45 \mu \mathrm{m}$ and having 74 frames per second). The systematic arrangement of polarization filters with orientations of $0^{\circ}, 45^{\circ}, 90^{\circ}$, and $135^{\circ}$ in the polarized camera is represented in the inset of Figure 2. The polarized camera records the raw intensity distribution of the hologram, and then simultaneously extracts the four multiple phase-shifted holograms without any spectral trade-offs.

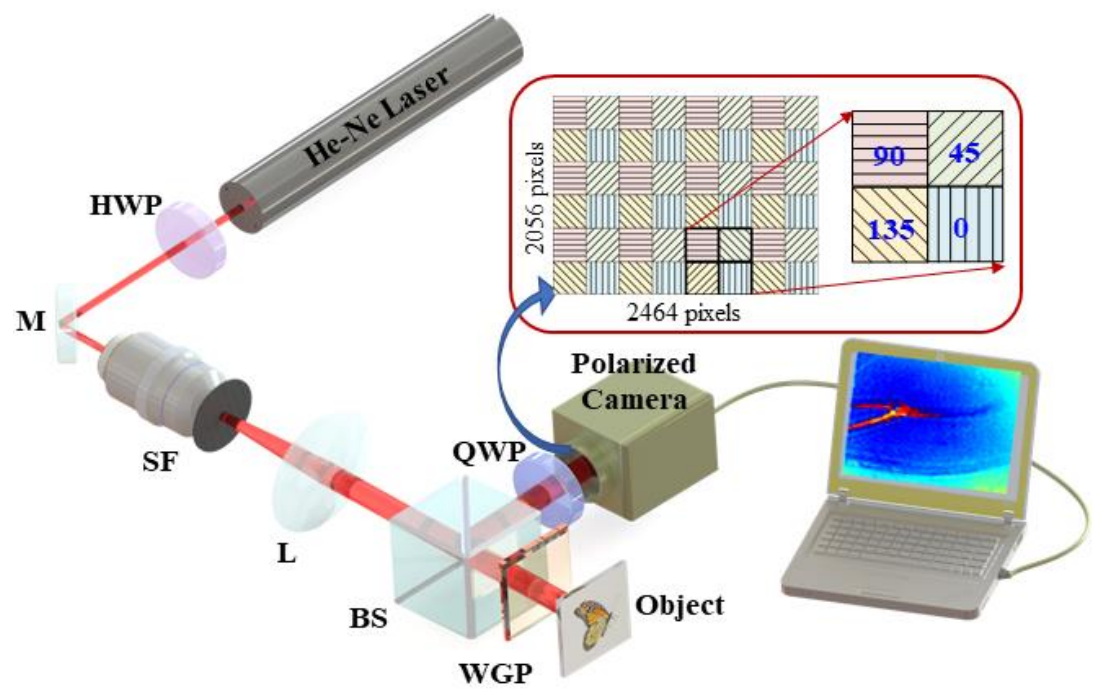

Figure 2. Experimental geometry of FP-PSDH: He-Ne laser: Helium-Neon laser source; HWP: Half Wave Plate; M: Mirror; SF: Spatial Filter assembly; L: Lens; BS: Nonpolarizing Beam Splitter; WGP: Wire Grid Polarizer; QWP: Quarter Wave Plate. In inset, the polarization filter arrangements in the polarized camera and respective orientation angles are represented. 


\section{Results and Discussion}

\subsection{Complex-Valued and Dynamic Object Imaging}

The performance and applicability of the FP-PSDH technique was experimentally tested for various objects and imaging conditions. To demonstrate the complex-valued imaging potential of the technique, we utilized the spatial light modulator (SLM) to introduce various complex-valued and pure phase objects. The complex-valued objects utilized for validating the technique were introduced using the phase-only SLM (PLUTO-VIS, Holoeye with total pixels $1920 \times 1080$, pixel pitch of $8 \mu \mathrm{m}$, and an image frame rate of $60 \mathrm{~Hz}$ ). A complex-valued object was designed to encode the SLM using the checkerboard method [36,37], in which the alternate pixels were assigned with a uniform binary phase value of 0 and $\pi$ (see Supplementary S1). We designed a complex object of Chinese characters 'Hua (华)' with an amplitude distribution and 'Da (大)' with a phase distribution, each consisting of size $3.9 \mathrm{~mm} \times 3.9 \mathrm{~mm}$ as shown in Figure 3a. The polarization camera recorded a single-shot hologram of the object, and the recorded intensity of the hologram is shown in Figure 3b. Consequently, the four phase-shifted holograms were extracted from the single-shot hologram, and the complex amplitude distribution of the object at the sensor plane was successfully retrieved from these phase-shifted holograms by utilizing Equation (4). The retrieved amplitude and phase distribution at the sensor plane are shown in Figures $3 c, d$, respectively. The recovery of the complex field distribution at the sensor plane using the phase-shifting technique provided the flexibility to reconstruct the object information at the desired plane using digital propagation based on the angular spectrum method [35]. The focused object information retrieved from the proposed technique is shown in Figure 3e,f. The amplitude distribution in Figure 3e shows a focused reconstruction of the amplitude '华' at the object plane and a blurred '大', as it is a pure phase distribution. In a similar way, Figure $3 f$ represents the focused phase distribution of the object, in which the phase part is reconstructed with good quality.

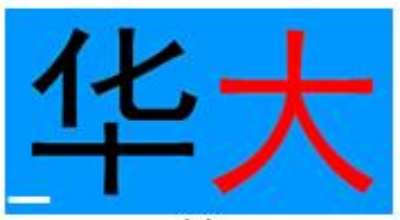

(a)

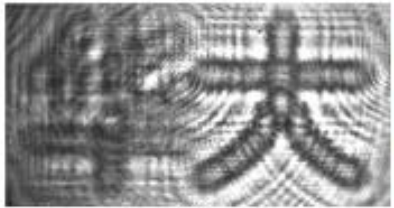

(b)

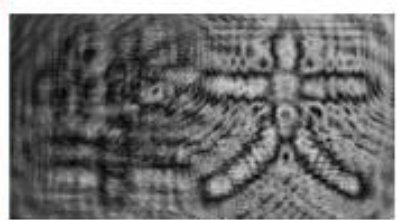

(c)

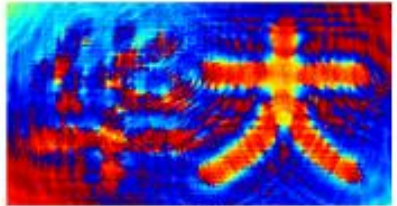

(d)

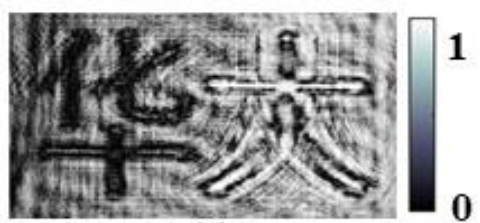

(e)

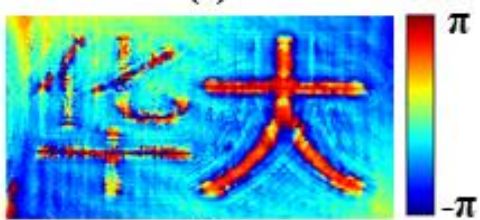

(f)

Figure 3. Complex field reconstruction results: (a) complex-valued object encoded in the SLM; (b) single-shot intensity of hologram recorded; (c) retrieved amplitude distribution at the sensor plane; (d) retrieved phase distribution at the sensor plane; (e) reconstructed amplitude distribution at the object plane; (f) reconstructed phase distribution at the object plane. Scale bar is $1.0 \mathrm{~mm}$.

Additionally, the potential of the technique is demonstrated for real-time imaging of dynamic sample by utilizing the single-shot imaging capability of the FP-PSDH. A dynamic phase object (running fox of size $7.2 \mathrm{~mm} \times 3.6 \mathrm{~mm}$ ) is designed and projected into the system using the SLM. A single-shot recording captures the hologram of the object in an instant, and further digital processing retrieves the four phase-shifted holograms. The phase object at the desired plane is reconstructed using the phase-shifting interferometry technique. To demonstrate the real time imaging of the moving target, we have recorded several intensity images in the sensor plane with a time interval of $0.1 \mathrm{~s}$. The reconstructed motion pictures at different instants of time of the moving phase object are shown in 
Figure 4. The reconstructed dynamic phase distribution of the object is presented in Visualization S1.

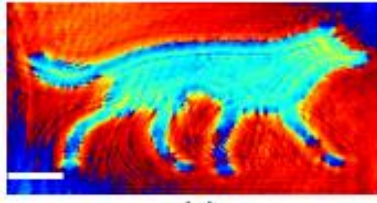

(a)

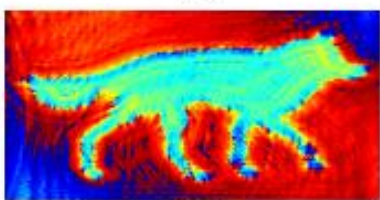

(d)

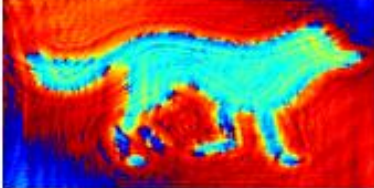

(b)

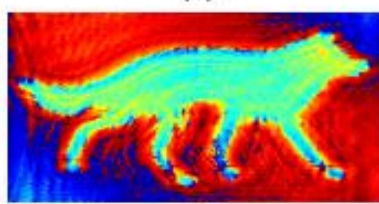

(e)

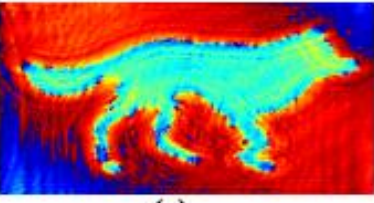

(c)

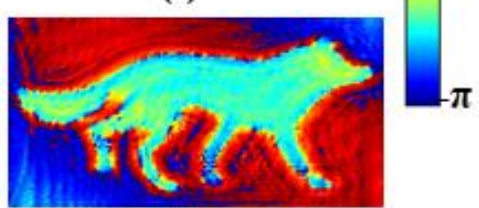

(f)

Figure 4. Experimental results for dynamic pure phase objects: (a-f) reconstructed motion picture phase distribution at the sensor plane for various instants of time. Scale bar is $0.86 \mathrm{~mm}$.

\subsection{Weakly Reflective Object Imaging}

Furthermore, we have investigated the image reconstruction quality of the FP-PSDH system for the case of real-world weakly reflective type objects. As the light reflection from these objects are comparatively weak, we integrated a 4f-imaging geometry along with the system to grab the light from the object surface to the sensor plane. Experiments were carried out for butterfly wings and a standard USAF negative (reflective) target, and the respective experimental results are shown in Figure 5. Figure 5a,d represent the single-shot raw intensity distribution of the recorded hologram corresponding to the butterfly wing and resolution test target, respectively. Subsequently, the multiple phase-shifted holograms were extracted from the single-shot recorded hologram, and the respective complex amplitude distribution of the object at the sensor plane was successfully retrieved from these multiple phase-shifted holograms using Equation (4). The reconstructed amplitude and phase distributions of butterfly wings is shown in Figures $5 b, c$, respectively. The phase distribution of butterfly wings shows a clear distinction between the discal cell and inner margin. In a similar way, the amplitude and phase distribution of the USAF resolution test target were reconstructed from digitally extracted multiple phase-shifted holograms, and the corresponding results are shown in Figures $5 \mathrm{e}, \mathrm{f}$, respectively. The system had a good resolving ability up to group 5 element 6 of the USAF resolution test target, which corresponds to 57.0 -line pairs $/ \mathrm{mm}$.

\subsection{Quantitative Analysis of System Stability and Sensitivity}

To evaluate the robustness of the system in environmental fluctuations and other noise mechanisms, we estimated the phase stability and sensitivity of the FP-PSDH system and compared it with other phase-shifting based methods. The performance was evaluated using time sequential detection of single-shot holograms in FP-PSDH, and it was compared with the two configurations of the Mach Zehnder interferometry (MZI) based phase-shifting scheme, namely multiple-shot phase-shifting MZI (MP-MZI) and single-shot polarization phase-shifting MZI (PP-MZI) (see Supplementary S1). In the case of FP-PSDH and PPMZI, the sequential detections of 50 single-shot holograms with a polarized camera in sample-free configuration were carried out with a time interval of $0.1 \mathrm{~s}$, and the phase map was recovered from respective digitally processed multiple phase shifted holograms. On the other hand, in the case of MP-MZI, the sequential detections of 50 holograms for each of the four phase-shifted holograms were recorded manually using a monochrome camera, and the respective phase was recovered. To evaluate the temporal stability, we estimated the phase fluctuation of a specific point in the recovered phase map with respect to the same point in the entire 50 recovered phase maps. The corresponding plot of the phase fluctuations with respect to the time sequential measurements is shown in Figure 6a. 
The estimation of standard deviation (STD) from the phase fluctuations shows that the proposed FP-PSDH technique has a lower STD (4.02 mRad) in comparison to MP-MZI (STD of $18.95 \mathrm{mRad}$ ) and PP-MZI (STD of $12.20 \mathrm{mRad}$ ). Furthermore, we also estimated the spatial sensitivity, which is the minimum detectable phase change in a recovered phase map for a particular measurement [38]. We evaluated the STD corresponding to total pixels in each of the 50 recovered phase maps for the respective phase-shifting based approaches. The box plot corresponding to all three configurations are shown in Figure $6 \mathrm{~b}$. The estimated STD for FP-PSDH is low in comparison to other techniques, which can be attributed to the high spatial sensitivity of the proposed technique. A quantitative comparison of the FP-PSDH system performance to MZI-based on-axis techniques is summarized in Table 1. The plots in Figure $6 \mathrm{a}, \mathrm{b}$ and the quantitative evaluation in Table 1 manifest the dominance of on-axis single-shot FP-PSDH in complex-valued dynamic object imaging over other techniques in the noise-assisted environments.
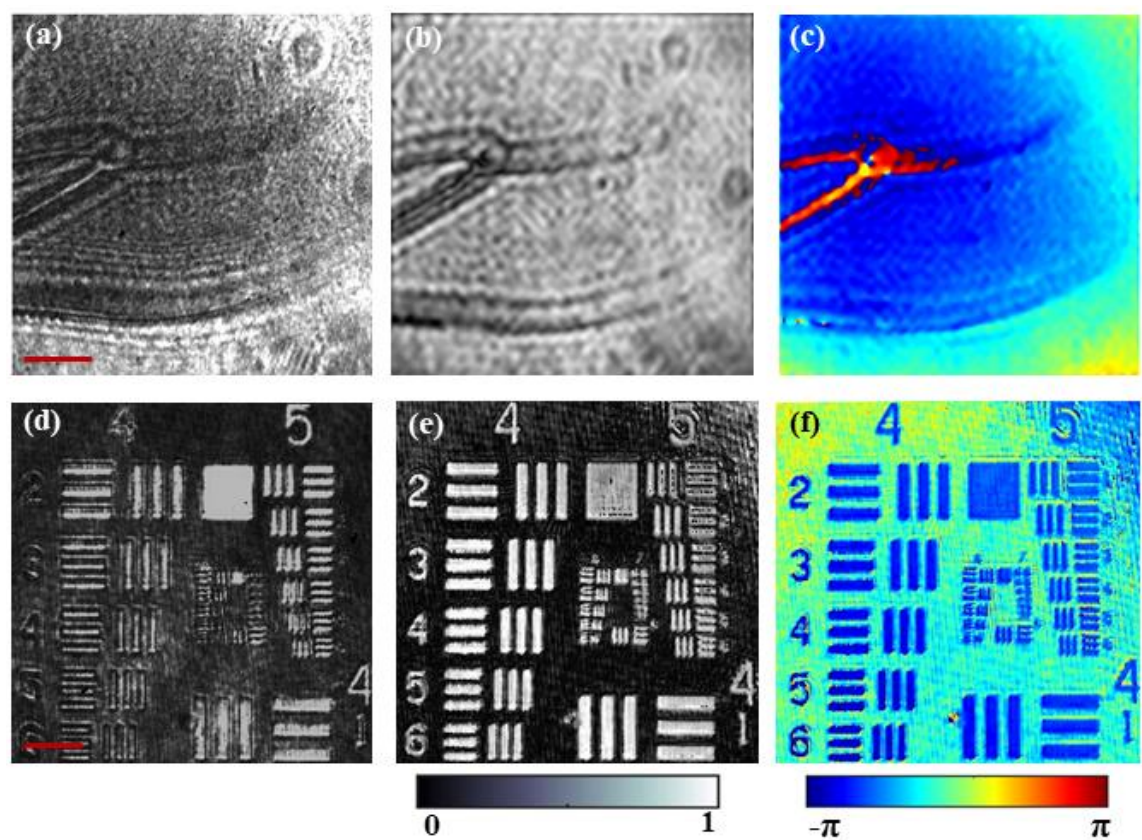

Figure 5. Experimental results: Butterfly wing; (a) raw intensity distribution of single-shot recorded hologram; (b) reconstructed amplitude distribution; (c) reconstructed phase distribution. USAF negative (reflective) target; (d) raw intensity distribution of single-shot recorded hologram; (e) reconstructed amplitude distribution; (f) reconstructed phase distribution. A 4f-imaging system with lenses of focal length $100 \mathrm{~mm}$ and $200 \mathrm{~mm}$ is utilized to image the object plane to the sensor plane. Scale bar: (a-c) $300 \mu \mathrm{m},(\mathbf{d}-\mathbf{f}) 100 \mu \mathrm{m}$.

Table 1. Quantitative comparison of FP-PSDH system performance with MP-MZI and PPMZI techniques.

\begin{tabular}{ccccc}
\hline $\begin{array}{c}\text { Interferometry } \\
\text { Scheme }\end{array}$ & $\begin{array}{c}\text { Type of } \\
\text { Geometry }\end{array}$ & $\begin{array}{c}\text { Detection } \\
\text { Scheme }\end{array}$ & $\begin{array}{c}\text { Temporal } \\
\text { Stability } \\
\text { (mRad) }\end{array}$ & $\begin{array}{c}\text { Spatial } \\
\text { Sensitivity } \\
\text { (mRad) }\end{array}$ \\
\hline MP-MZI & double path & four-shot & 18.95 & 27.76 \\
PP-MZI & double path & single-shot & 12.20 & 24.86 \\
FP-PSDH & single path & single-shot & 4.02 & 17.47 \\
\hline
\end{tabular}



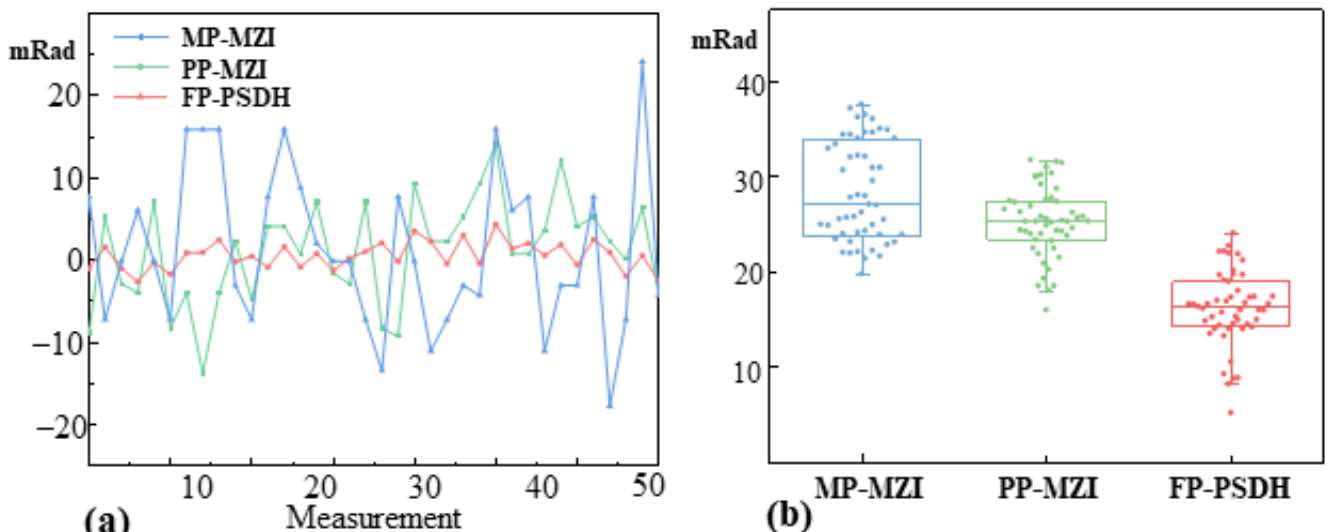

(a)

(b)

Figure 6. Quantitative evaluation of system stability and sensitivity: (a) Temporal stability evaluation of MP-MZI, PP-MZI, and FP-PSDH. The X-axis represents the time sequential measurements, and the $\mathrm{Y}$-axis represents the respective phase fluctuations for a specific point in the recovered phase map; (b) Spatial sensitivity evaluation of MP-MZI, PP-MZI, and FP-PSDH with the respective box plots. In each box plot, the lower and upper boundaries represent the first and third quartiles, respectively, and the line in each box represents the median value.

\section{Conclusions}

In conclusion, we have developed a single-shot on-axis PSDH technique based on a Fizeau polarization interferometry approach for complex-valued static and dynamic object imaging. The adoption of the space division multiplexing along with the utilization of a polarized camera provides the potential realization in the development of a highly stable real-time complex field imaging system. The effectiveness of the technique is experimentally demonstrated by imaging different kinds of reflecting-type complex-valued samples. The compact design of FP-PSDH with on-axis geometry exhibits the dominance of the proposed technique over conventional phase-shifting techniques for the imaging of dynamic events. Moreover, the near common-path single-shot phase-shifting technique utilized in the FP-PSDH system enhances the space-bandwidth in comparison to the existing off-axis digital holography systems with its unique compact on-axis design. In addition, the highstability feature resulting from on-axis interferometry geometry provides the possible integration of an optical microscopy system for the development of a robust quantitative phase microscopy system for dynamic phase measurements.

Supplementary Materials: The following are available online at https:/ /www.mdpi.com/article/10 .3390 / photonics9030126/s1, The digital reconstruction scheme (Figure S1), complex-valued object details (Figure S2), experimental design of MZI scheme (Figure S3), and the dynamic phase object visualization $\mathrm{S} 1$.

Author Contributions: Conceptualization, V.R.V.; methodology, H.L. and V.R.V.; formal analysis, H.R. and V.R.V.; investigation, H.L., X.D., and V.R.V.; data curation, H.L. and V.R.V.; writing-original draft preparation, H.L. and V.R.V.; writing—review and editing, V.R.V., Z.C., and J.P.; supervision, J.P.; funding acquisition, Z.C. and V.R.V. All authors have read and agreed to the published version of the manuscript.

Funding: This work was supported by the Natural Science Foundation of China (NSFC) under Grants No. 11674111, No. 62005086, and No. 12150410318.

Institutional Review Board Statement: Not applicable.

Informed Consent Statement: Not applicable.

Data Availability Statement: The data that support the findings of this study are available from thecorresponding author upon request.

Conflicts of Interest: The authors declare no conflict of interest. 


\section{References}

1. Kreis, T. Handbook of Holographic Interferometry; Wiley-VCH: Weinheim, Germany, 2004.

2. Tahara, T.; Quan, X.; Otani, R.; Takaki, Y.; Matoba, O. Digital holography and its multidimensional imaging applications: A review. Microscopy 2018, 67, 55-67. [CrossRef]

3. Paturzo, M.; Pagliarulo, V.; Bianco, V.; Memmolo, P.; Miccio, L.; Merola, F.; Ferraro, P. Digital Holography, a metrological tool for quantitative analysis: Trends and future applications. Opt. Lasers Eng. 2018, 104, 32-47. [CrossRef]

4. Balasubramani, V.; Kujawińska, M.; Allier, C.; Anand, V.; Cheng, C.-J.; Depeursinge, C.; Hai, N.; Juodkazis, S.; Kalkman, J.; Kuś, A.; et al. Roadmap on Digital Holography-Based Quantitative Phase Imaging. J. Imaging 2021, 7, 252. [CrossRef] [PubMed]

5. Javidi, B.; Carnicer, A.; Anand, A.; Barbastathis, G.; Chen, W.; Ferraro, P.; Goodman, J.; Horisaki, R.; Khare, K.; Kujawinska, M.; et al. Roadmap on digital holography [Invited]. Opt. Express 2021, 29, 35078. [CrossRef] [PubMed]

6. Chen, L.; Singh, R.K.; Vinu, R.V.; Chen, Z.; Pu, J. A wavefront division multiplexing holographic scheme and its application in looking through diffuser. New J. Phys. 2021, 23, 113034. [CrossRef]

7. Chen, L.; Chen, Z.; Singh, R.K.; Vinu, R.; Pu, J. Increasing field of view and signal to noise ratio in the quantitative phase imaging with phase shifting holography based on the Hanbury Brown-Twiss approach. Opt. Lasers Eng. 2021, 148, 106771. [CrossRef]

8. Vinu, R.V.; Chen, Z.; Singh, R.K.; Pu, J. Ghost diffraction holographic microscopy. Optica 2020, 7, 1697. [CrossRef]

9. Xu, W.; Jericho, M.H.; Meinertzhagen, I.A.; Kreuzer, H.J. Digital in-line holography for biological applications. Proc. Natl. Acad. Sci. USA 2001, 98, 11301-11305. [CrossRef]

10. Cuche, E.; Bevilacqua, F.; Depeursinge, C. Digital holography for quantitative phase-contrast imaging. Opt. Lett. 1999, 24, $291-293$. [CrossRef]

11. Osten, W.; Faridian, A.; Gao, P.; Körner, K.; Naik, D.; Pedrini, G.; Singh, A.K.; Takeda, M.; Wilke, M. Recent advances in digital holography [Invited]. Appl. Opt. 2014, 53, G44-G63. [CrossRef]

12. Hai, N.; Rosen, J. Coded aperture correlation holographic microscope for single-shot quantitative phase and amplitude imaging with extended field of view. Opt. Express 2020, 28, 27372. [CrossRef]

13. Kumar, M.; Quan, X.; Awatsuji, Y.; Cheng, C.; Hasebe, M.; Tamada, Y.; Matoba, O. Common-path multimodal three-dimensional fluorescence and phase imaging system. J. Biomed. Opt. 2020, 25, 1. [CrossRef]

14. Latychevskaia, T.; Fink, H.-W. Solution to the Twin Image Problem in Holography. Phys. Rev. Lett. 2007, 98, 233901. [CrossRef]

15. Monaghan, D.S.; Kelly, D.P.; Pandey, N.; Hennelly, B.M. Twin removal in digital holography using diffuse illumination. Opt. Lett. 2009, 34, 3610-3612. [CrossRef]

16. Anand, V.; Katkus, T.; Linklater, D.P.; Ivanova, E.P.; Juodkazis, S. Lensless Three-Dimensional Quantitative Phase Imaging Using Phase Retrieval Algorithm. J. Imaging 2020, 6, 99. [CrossRef]

17. Rivenson, Y.; Wu, Y.; Ozcan, A. Deep learning in holography and coherent imaging. Light. Sci. Appl. 2019, 8, 85. [CrossRef]

18. Li, H.; Chen, X.; Chi, Z.; Mann, C.; Razi, A. Deep DIH: Single-Shot Digital In-Line Holography Reconstruction by Deep Learning. IEEE Access 2020, 8, 202648-202659. [CrossRef]

19. Yamaguchi, I.; Zhang, T. Phase-shifting digital holography. Opt. Lett. 1997, 22, 1268-1270. [CrossRef]

20. Yamaguchi, I.; Kato, J.-I.; Ohta, S.; Mizuno, J. Image formation in phase-shifting digital holography and applications to microscopy. Appl. Opt. 2001, 40, 6177-6186. [CrossRef]

21. Awatsuji, Y.; Sasada, M.; Kubota, T. Parallel quasi-phase-shifting digital holography. Appl. Phys. Lett. 2004, 85, 1069-1071. [CrossRef]

22. Awatsuji, Y.; Tahara, T.; Kaneko, A.; Koyama, T.; Nishio, K.; Ura, S.; Kubota, T.; Matoba, O. Parallel two-step phase-shifting digital holography. Appl. Opt. 2008, 47, D183-D189. [CrossRef] [PubMed]

23. Lin, M.; Nitta, K.; Matoba, O.; Awatsuji, Y. Parallel phase-shifting digital holography with adaptive function using phase-mode spatial light modulator. Appl. Opt. 2012, 51, 2633-2637. [CrossRef] [PubMed]

24. Awatsuji, Y.; Kakue, T.; Tahara, T.; Xia, P.; Nishio, K.; Ura, S.; Kubota, T.; Matoba, O. Parallel phase-shifting digital holography system using a high-speed camera. In Proceedings of the Optical Design and Testing V, Photonics Asia, Beijing, China; 2012; Volume 8557, p. 85570E.

25. Wang, D.; Liang, R. Simultaneous polarization Mirau interferometer based on pixelated polarization camera. Opt. Lett. 2015, 41, 41-44. [CrossRef] [PubMed]

26. Ishikawa, K.; Tanigawa, R.; Yatabe, K.; Oikawa, Y.; Onuma, T.; Niwa, H. Simultaneous imaging of flow and sound using high-speed parallel phase-shifting interferometry. Opt. Lett. 2018, 43, 991-994. [CrossRef] [PubMed]

27. Yatabe, K.; Tanigawa, R.; Ishikawa, K.; Oikawa, Y. Time-directional filtering of wrapped phase for observing transient phenomena with parallel phase-shifting interferometry. Opt. Express 2018, 26, 13705-13720. [CrossRef]

28. Tian, X.; Liang, R. Snapshot phase-shifting diffraction phase microscope. Opt. Lett. 2020, 45, 3208-3211. [CrossRef]

29. Tahara, T.; Kanno, T.; Arai, Y.; Ozawa, T. Single-shot phase-shifting incoherent digital holography. J. Opt. 2017, 19, 065705. [CrossRef]

30. Tsuruta, M.; Fukuyama, T.; Tahara, T.; Takaki, Y. Fast Image Reconstruction Technique for Parallel Phase-Shifting Digital Holography. Appl. Sci. 2021, 11, 11343. [CrossRef]

31. Tahara, T.; Kozawa, Y.; Oi, R. Single-path single-shot phase-shifting digital holographic microscopy without a laser light source. Opt. Express 2022, 30, 1182. [CrossRef] 
32. Fukuda, T.; Awatsuji, Y.; Xia, P.; Kakue, T.; Nishio, K.; Matoba, O. Review of three-dimensional imaging of dynamic objects by parallel phase-shifting digital holography. Opt. Eng. 2018, 57, 061613. [CrossRef]

33. Fukuda, T.; Shinomura, M.; Xia, P.; Awatsuji, Y.; Nishio, K.; Matoba, O. Three-dimensional motion-picture imaging of dynamic object by parallel-phase-shifting digital holographic microscopy using an inverted magnification optical system. Opt. Rev. 2017, 24, 206-211. [CrossRef]

34. Xia, P.; Ri, S.; Inoue, T.; Awatsuji, Y.; Matoba, O. Dynamic phase measurement of a transparent object by parallel phase-shifting digital holography with dual polarization imaging cameras. Opt. Lasers Eng. 2021, 141, 106583. [CrossRef]

35. Goodman, J.W. Introduction to Fourier Optics; McGraw-Hill: New York, NY, USA, 1996.

36. Dudley, A.; Vasilyeu, R.; Belyi, V.; Khilo, N.; Ropot, P.; Forbes, A. Controlling the evolution of nondiffracting speckle by complex amplitude modulation on a phase-only spatial light modulator. Opt. Commun. 2012, 285, 5-12. [CrossRef]

37. Ulusoy, E.; Onural, L.; Ozaktas, H.M. Full-complex amplitude modulation with binary spatial light modulators. J. Opt. Soc. Am. A 2011, 28, 2310-2321. [CrossRef]

38. Hai, N.; Rosen, J. Single-plane and multiplane quantitative phase imaging by self-reference on-axis holography with a phaseshifting method. Opt. Express 2021, 29, 24210. [CrossRef] 